\title{
Role of Phosphorylation in the Mammalian Circadian Clock
}

\author{
K. VANSELOW AND A. KRAMER \\ Laboratory of Chronobiology, Charité Universitätsmedizin Berlin, \\ 10115 Berlin, Germany
}

\begin{abstract}
Circadian clocks regulate a wide variety of processes ranging from gene expression to behavior. At the molecular level, circadian rhythms are thought to be produced by a set of clock genes and proteins interconnected to form transcriptional-translational feedback loops. Rhythmic gene expression was formerly regarded as the major drive for rhythms in clock protein abundance, but recent findings underline the crucial importance of posttranslational mechanisms for both the generation and dynamics of circadian rhythms. In particular, the reversible phosphorylation of PER proteins - essential components within the negative feedback loop in Drosophila and mammals - seems to have a key role for the correct timing of nuclear repression. To understand how PER protein phosphorylation regulates the dynamics of the circadian oscillator, we have mapped endogenous phosphorylation sites in mPER2. Detailed investigation of the functional role of one particular phosphorylation site (Ser-659, which is mutated in the familial advanced sleep phase syndrome [FASPS]) led us propose a model of functionally different phosphorylation sites in PER2. This concept explains not only the FASPS phenotype, but also the effect of the tau mutation in hamster.
\end{abstract}

\section{INTRODUCTION}

Most light-sensitive organisms have evolved internal clocks that regulate daily rhythms in physiology, metabolism, and behavior. In a natural environment, these clocks are synchronized to external zeitgebers, such as the light/dark cycle or temperature cycles, to ensure a stable phase relationship between internal and external processes. Under constant conditions, oscillations are freerunning with a period close to 24 hours, hence the term circa-dian clocks. Extensive studies in the last two decades have unraveled the molecular basis of circadian rhythmicity in a broad variety of organisms such as cyanobacteria, plants, Neurospora, Drosophila, and mammals. Conceptually, circadian rhythms are generated by delayed negative feedback loops within single cells. A set of so-called clock genes and clock proteins are interconnected to produce self-sustained circadian oscillations at the molecular level.

In mammals, a negative gene-regulatory feedback loop is at the heart of the circadian oscillator: CLOCK and BMAL1-bHLH (basic helix-loop-helix)-PAS (PeriodArnt-Single-minded)-containing transcription factorsheterodimerize and bind to E-box enhancer elements in the promoter region of the Period (Per1, Per2) and Cryptochrome (Cry1, Cry2) genes to activate their transcription. PER and CRY proteins, together with casein kinase $1 \varepsilon / \delta(\mathrm{CKI} \varepsilon / \delta)$ and probably other proteins, form a large multimeric complex, the circadian feedback module (Brown et al. 2005a; Hofmann et al. 2006). It is believed that after some time, the complex enters the nucleus, where PER and CRY proteins inhibit their own synthesis by directly interacting with the CLOCK-BMAL1 heterodimer. At present, the exact composition of the module is unclear, although multiple additional proteins have been suggested to contribute to the periodic inhibition of CLOCK-BMAL1 activity. These include mammalian Timeless (Sangoram et al. 1998; Barnes et al. 2003), the
bHLH transcription factors DEC1/2 (Honma et al. 2002), the PER1-associated proteins NONO and WDR5 (Brown et al. 2005a) as well as the CLOCK-interacting protein CIPC (Zhao et al. 2007).

Further feedback loops possibly contribute to the robustness of the system. In a positive feedback loop, the transcription of the Bmall gene is regulated by rhythmic action of Rev-Erbo, the transcription of which is also controlled by CLOCK-BMAL1 via E-box elements (Preitner et al. 2002).

Critical for the generation of a self-sustained circadian oscillation with a period of about 24 hours is a time delay of several hours between the synthesis of PER and CRY proteins and their action as inhibitors of their own expression in the nucleus. Several mechanisms are discussed to participate in the generation of this delay. These mechanisms modulate abundance, localization, and activity of transcriptional inhibitors by regulating transcription, translation, and posttranslational modifications of important components of the circadian oscillator.

Up to now, many posttranslational mechanisms have been discovered to modulate circadian dynamics, although we are far from having a comprehensive view on the function of posttranslational modifications for the clock. Perhaps the most important posttranslational modification in eukaryotic cells is the phosphorylation of serine, threonine, and tyrosine residues. Such phosphorylations frequently act as molecular switches between active and inactive protein states either directly by regulating the activity (e.g., kinases) or indirectly by altering subcellular localization (e.g., transcription factors). In addition, phosphorylation often initiates protein degradation by the proteasome. Target proteins contain specific amino acid motifs, which, when phosphorylated, form a recognition sequence for the ubiquitin ligase complex.

Several important components of the mammalian circadian oscillator are known to be targets of posttranslational modifications. CLOCK, BMAL1, PER, and 
possibly also CRY proteins are phosphoproteins in vivo (Lee et al. 2001). Moreover, BMAL1 was shown to be sumoylated (Cardone et al. 2005) and, very recently, acetylated (Grimaldi et al., this volume). These modifications specifically alter the properties and functionality of the corresponding proteins by changing protein stability, subcellular localization, activity, or complex formation.

Hitherto, phosphorylation is the best-explored posttranslational mechanism within essentially all circadian model systems. Recent results from cyanobacteria even challenge the dogma of rhythmic transcriptional-translational feedback loops as the fundamental principle of rhythm generation: A purely posttranslational mechanism based on rhythmic phosphorylation and dephosphorylation of the hexameric KaiC protein is sufficient to generate and sustain circadian rhythms at least in vitro (Nakajima et al. 2005; Tomita et al. 2005). In flies and mammals, there is also evidence for the particular importance of posttranslational mechanisms: Constitutive expression of $d P e r$ in arrythmic $p^{0} r^{0}$ flies rescues arrhythmic behavior and leads to rhythmic PER protein levels (Yang and Sehgal 2001). Similarly, constitutive expression of mammalian Per genes in synchronized cultured cells also results in rhythmic PER protein abundance (Fujimoto et al. 2006; Nishii et al. 2006). Consequently, these data suggest that rhythmic PER protein abundance in vivo might be generated by a combination of rhythmic transcription of $\mathrm{Per}$ genes and posttranslational events. At least in the case of flies, the posttranslational mechanism seems to be sufficient for behavioral circadian rhythmicity.

This chapter focuses on the functional role of clock protein phosphorylation for the generation and the dynamics of circadian oscillations with a special emphasis on mammalian and Drosophila PER proteins.

\section{Kinases and Phosphatases}

Doubletime (DBT) - the Drosophila homolog of mammalian CKIE - was the first kinase identified to have an important functional role in the circadian system of Drosophila. Flies with mutations in the $d b t$ gene have altered circadian periods at both the behavioral and molecular levels. In $d b t$ mutant flies, the stability, phosphorylation pattern, and subcellular localization of dPER is modified (Kloss et al. 1998; Price et al. 1998). Subsequent studies showed an additional role for DBT in the phosphorylation of dCLOCK, thereby regulating its stability (Kim and Edery 2006; Yu et al. 2006). In addition, Drosophila casein kinase 2 (dCK2) was shown to phosphorylate dPER. This phosphorylation predominantly functions in the regulation of the subcellular localization of dPER by promoting its nuclear entry and accumulation, whereas its effects on dPER stability are rather weak (Lin et al. 2002, 2005; Akten et al. 2003). Moreover, Shaggy - the Drosophila homolog of mammalian glycogen synthase kinase $3 \beta$ (GSK3 $\beta$ ) — phosphorylates dTIM, the essential binding partner of dPER, thereby promoting the nuclear entry of the PER/TIM heterodimer (Martinek et al. 2001). The impact of Shaggy phosphorylation on TIM stability has not yet been eluci- dated, although it has been reported that phosphorylated TIM species are more prone to light-induced proteasomal degradation (Zeng et al. 1996).

The discovery of DBT's importance in the Drosophila circadian system initiated extensive studies to elucidate a possible conserved function of the DBT homologous kinases of the CKI family in the mammalian clockwork. Indeed, it has been demonstrated that CKI $\varepsilon$ and CKI $\delta$ are involved in the phosphorylation of the mammalian PER proteins (Keesler et al. 2000; Camacho et al. 2001; Akashi et al. 2002; Schlosser et al. 2005). Moreover, CKIE is able to phosphorylate BMAL1 and CRY proteins in vitro (Eide et al. 2002).

Recent studies showed that GSK3 $\beta$ also has a role in the mammalian circadian system, but interestingly, its targets do not seem to be conserved. Whereas Shaggy phosphorylates dTIM in Drosophila, substrates of mammalian GSK3 $\beta$ are CRY2 (Harada et al. 2005), Rev-erb $\alpha$ (Yin et al. 2006) and PER2 (Iitaka et al. 2005). Here, GSK3 $\beta$-mediated phosphorylation regulates the stability of CRY2 and Rev-erbo as well as the subcellular localization of PER2.

It is still unclear whether additional kinases are involved in the generation of circadian rhythmicity in mammals. However, at least in the case of mPER2, it has been speculated that yet unknown kinases are involved in phosphorylation-dependent subcellular localization (Vanselow et al. 2006).

Phosphatases are the natural opponents of kinases: They ensure reversibility of phosphorylation-induced alterations in protein function. The role of phosphatases in the circadian clockwork is just emerging. So far, it has been demonstrated in Drosophila that PP2A dephosphorylates and thus stabilizes dPER (Sathyanarayanan et al. 2004). In mammals, PP1 was shown to dephosphorylate a mPER2 fragment in vitro. In addition, coexpression with a dominant-negative version of PP1 destabilizes this mPER2 fragment, indicating that dephosphorylation of PER proteins by PP1 may counteract $\mathrm{CKI} \varepsilon / \delta$-induced proteasomal degradation (Gallego et al. 2006a). Furthermore, PP5 has been reported to regulate the kinase activity of CKIE by antagonizing its inhibitory autophosphorylation. Interestingly, the PP5-mediated activation of CKI $\varepsilon$ is inhibited by cryptochrome proteins (Partch et al. 2006), which may provide an explanation for the stabilizing effect of CRYs on PER proteins.

\section{PER Phosphorylation}

In the 1990s, a delayed negative feedback loop was proposed as the fundamental principle of circadian rhythm generation in Drosophila. This was primarily based on the striking 4-6-hour lag between the accumulation profiles of Drosophila Per mRNA and protein (Hardin et al. 1990), leading to speculations that a pure transcriptional mechanism is not sufficient to create this delay. When analyzing the circadian accumulation patterns of dPER and mammalian PERs by western blotting, a substantial electrophoretic mobility shift of the PER bands occurred, in addition to a high-amplitude variation in protein abundance. This mobility shift has been shown 


\section{PHOSPHORYLATION IN THE MAMMALIAN CIRCADIAN CLOCK}

to be due to a gradual, circadian phase-dependent phosphorylation of the PER proteins (Edery et al. 1994; Lee et al. 2001). Interestingly, the timing and extent of the PER phosphorylation are modified in period-altering fly mutants (Edery et al. 1994), indicating that the phosphorylation status of the PER proteins reflects the progress in the circadian cycle.

Several properties of PER proteins turned out to be directly regulated by the degree of PER phosphorylation, such as stability, subcellular localization, and inhibitory activity (see below). Importantly, the phosphorylation of PER proteins in Drosophila and mammals is not mediated by a single kinase but is the result of a complex temporal and spatial interplay of several kinases and phosphatases.

Phosphorylation regulates stability. Already in one of the first studies on Drosophila PER protein, it has been noted that the dPER species with the highest degree of phosphorylation are present at times right before the drastic drop of protein abundance. This suggested a direct link between dPER phosphorylation and degradation (Edery et al. 1994). In $d b t^{P}$ larvae (a $d b t$ loss-of-function mutant), dPER is hypophosphorylated and accumulates to unusual high levels (Price et al. 1998), further indicating that phosphorylation destabilizes PER. Subsequently, it was shown that progressive phosphorylation of dPER triggers the binding of the F box/WD40-repeat protein Slimb. Slimb functions as a substrate-recognizing component of the ubiquitin ligase SCF complex and promotes the ubiquitination and subsequent proteasomal degradation of hyperphosphorylated PER (Grima et al. 2002; Ko et al. 2002). As slimb mutant flies are behaviorally arrhythmic, the time-specific degradation of PER proteins is an essential part of circadian rhythm generation and maintenance. It adds to the required time delay within the negative feedback loop by counteracting a premature cytoplasmic and nuclear accumulation of the inhibitory complex.

In a very similar manner, mammalian PER proteins are extensively phosphorylated by CKI $\varepsilon$ and CKI $\delta$, thereby reducing the stability of the proteins by targeting them for proteasomal degradation (Keesler et al. 2000; Camacho et al. 2001; Akashi et al. 2002). PER proteins are recognized by the Slimb homologs $\beta$-TrCP $1 / 2$ as parts of the SCF ubiquitin ligase complex. The interactions between $\beta$ TrCP1/2 and their substrates occur via specific recognition sequences within the substrate. For both mPER2 and mPER1, a $\beta$-TrCP1/2-binding site has been proposed (Eide et al. 2005; Shirogane et al. 2005). Down-regulation of both endogenous $\beta$-TrCP1 and $\beta$-TrCP2 in synchronized fibroblasts results in a substantial lengthening of the circadian period. Furthermore, the expression of $\beta$ TrCP1/2 interaction-deficient PER2 variants in synchronized fibroblasts leads to a dramatic stabilization of PER2 protein as well as to a disruption of circadian rhythms (Reischl 2007).

In mammals, PER proteins are not the only clock components, whose stability is controlled by the proteasomal pathway. Very recently, the F-box protein Fbxl3 was shown to target CRY proteins for proteasomal degradation. Mice with mutations in Fbxl3 display a lengthened circadian locomotor activity rhythm, an increased stabil- ity of the CRY proteins, and a reduced expression of PER proteins (Busino et al. 2007; Godinho et al. 2007; Siepka et al. 2007). Given the relatively high CRY protein amounts as well as the rather small amplitude of CRY protein abundance rhythms, the molecular basis of Fbxl3's essential role in the circadian clock is still, at least in part, obscure. For example, it is unclear whether phosphorylation of CRY proteins is required for recognition by Fbxi3.

Phosphorylation regulates subcellular localization. Phosphorylation affects not only PER protein stability, but also its subcellular localization. For example, in $d b t$ and per mutant flies, besides the accumulation profile, the nuclear import and nuclear clearance patterns of dPER are altered. Furthermore, Cyran et al. (2005) showed that phosphorylation by DBT retains PER in the cytoplasm, whereas hypophosphorylated dPER is able to enter the nucleus in $\mathrm{tim}^{01}$; $d b t^{P}$ double-mutant larvae and $\mathrm{tim}^{01}$; $d b t^{a r}$ double-mutant flies, respectively. Thus, proper dPER phosphorylation by DBT is crucial for correct timing of dPER nuclear entry and export. Besides DBT, the phosphorylation of CK2 has a clear impact on the subcellular localization of dPER. Mutations in the catalytic $\alpha$ or regulatory $\beta$ subunit of CK2 lengthen the circadian period of flies and concomitantly decelerate the nuclear entry of the PER protein (Lin et al. 2002; Akten et al. 2003). A similar role for CK2 has also been established in the Neurospora and Arabidopsis clock (Sugano et al. 1999; Yang et al. 2002). Given this striking conservation in several phylogenetic kingdoms, an important function for CK2 has been proposed in the mammalian system, but not yet established.

In mammals, the influence of CKI $/ \delta$ phosphorylation on the subcellular localization of PER proteins is still not fully understood. Several independent studies have addressed this issue with ambiguous and sometimes contradictory results. In cultured cells, the subcellular distribution of mammalian PER proteins following coexpression with CKI $\varepsilon$ or CKI $\delta$ seems to depend on the respective PER paralog as well as on the cell line. Although a direct effect of CKI $\varepsilon / \delta$ phosphorylation on subcellular localization can be detected, a consistent direction of this effect is not observed (Takano et al. 2000, 2004; Vielhaber et al. 2000; Akashi et al. 2002).

Phosphorylation regulates inhibitory activity. The activity of many eukaryotic transcription factors (e.g., NFAT, p53, HSF1, CREB, c-Jun, and Fos) is regulated by reversible phosphorylation (Holmberg et al. 2002). As PER proteins are believed to act as transcriptional repressors, it is conceivable that their inhibitory action on CLOCK:BMAL1 could be regulated by phosphorylation, as well. However, direct evidence for this is rare. Only one study demonstrates that phosphorylation of dPER by DBT and CK2 acts in a coordinate manner to potentiate PER repression activity. Importantly, this effect is not mediated by an alteration of the subcellular localization of the protein (Nawathean and Rosbash 2004). Similar results have not been reported in the mammalian system. 


\section{Circadian Phenotypes with Altered PER Phosphorylation}

How important is the phosphorylation of PER proteins for the generation and dynamics of circadian rhythms? Probably very important, because several severe circadian phenotypes are due to mutations that alter the temporal and spatial phosphorylation patterns of PER proteins. Examples for known circadian phenotypes with altered PER phosphorylation in Drosophila and mammals are summarized in Table 1 and described in more detail below.

The first direct evidence of a genetic basis for circadian clocks came from the short- and long-period mutant flies ( $\mathrm{per}^{S}$ and $\mathrm{per}^{L}$ ) isolated by Konopka and Benzer (1971). Later, it was shown that in per $^{S}$ mutants, the accumulation profile and nuclear clearance of dPER are advanced (Zerr et al. 1990; Edery et al. 1994), whereas in $p^{2} r^{L}$ flies, the nuclear entry of the PER protein is delayed (Curtin et al. 1995). Similar effects were observed in $d b t$ mutants (e.g., $d b t^{S}$ and $d b t^{L}$ ) (Kloss et al. 1998; Price et al. 1998).

In the mammalian circadian system, the most prominent examples of phosphorylation-based circadian phenotypes are the tau mutation in the hamster and the FASPS phenotype in humans. The tau mutation was discovered by Ralph and Menaker (1988) on the basis of a prominent shortening of the circadian locomotor activity rhythm-a 22-hour period in heterozygous animals and a 20-hour period in homozygous animals (Ralph and Menaker 1988). The mRNA profiles of Per 1 and Per2 in the SCN (suprachiasmatic nucleus) of tau mutant hamsters show an earlier rise and decline and a reduction in the peak expression levels of both genes (Lowrey et al. 2000; Dey et al. 2005). Genetic analysis revealed that a singlenucleotide exchange in the coding region of the CKIE gene generates the tau mutant phenotype. This mutation

Table 1. Circadian Phenotypes with Altered PER Phosphorylation in Drosophila and Mammals

\begin{tabular}{|c|c|c|c|c|c|}
\hline Locus/species & $\begin{array}{l}\text { Mutant } \\
\text { allele }\end{array}$ & $\begin{array}{l}\text { Molecular } \\
\text { lesion }\end{array}$ & Phenotype & Molecular impact & References \\
\hline $\begin{array}{l}\text { period gene, } \\
\text { Drosophila }\end{array}$ & $p_{e r}{ }^{S}$ & $\begin{array}{l}\text { single-nucleotide } \\
\text { exchange leads } \\
\text { to Ser-589 } \rightarrow \text { Asn }\end{array}$ & $\begin{array}{l}\text { short-period rhythms } \\
\text { (19 hr) in eclosion } \\
\text { and behavior }\end{array}$ & $\begin{array}{l}\text { abundance and phosphoryl- } \\
\text { ation profiles of PER }{ }^{\mathrm{S}} \\
\text { are advanced; premature } \\
\text { nuclear clearance, but } \\
\text { unaltered nuclear } \\
\text { accumulation }\end{array}$ & $\begin{array}{l}\text { Konopka and } \\
\text { Benzer (1971); } \\
\text { Yu et al. (1987); } \\
\text { Zerr et al. (1990); } \\
\text { Curtin et al. } \\
\text { (1995) }\end{array}$ \\
\hline $\begin{array}{l}\text { period gene, } \\
\text { Drosophila }\end{array}$ & $p e r^{L}$ & $\begin{array}{l}\text { single-nucleotide } \\
\text { exchange leads } \\
\text { to Val-243 } \rightarrow \text { Asp }\end{array}$ & $\begin{array}{l}\text { long-period rhythms } \\
(29 \mathrm{hr}) \text { in eclosion } \\
\text { and behavior }\end{array}$ & $\begin{array}{l}\text { nuclear entry and clearance } \\
\text { of } P^{2} R^{L} \text { is delayed }\end{array}$ & $\begin{array}{l}\text { Konopka and } \\
\text { Benzer (1971); } \\
\text { Curtin et al. } \\
\text { (1995) }\end{array}$ \\
\hline
\end{tabular}

\begin{tabular}{|c|c|c|c|}
\hline $\begin{array}{l}\text { doubletime } \\
\text { gene, } \\
\text { Drosophila }\end{array}$ & $d b t^{s}$ & $\begin{array}{l}\text { single-nucleotide } \\
\text { exchange leads to } \\
\text { Pro- } 47 \rightarrow \text { Ser }\end{array}$ & $\begin{array}{l}\text { short-period rhythms } \\
\text { in eclosion }(20 \mathrm{hr}) \\
\text { and behavior }(18 \mathrm{hr}\end{array}$ \\
\hline
\end{tabular}

$\begin{gathered}\text { doubletime } \\ \text { gene, }\end{gathered} d b t^{L}$
single-nucleotide
exchange leads

Drosophila $\quad$ to Met- $80 \rightarrow$ Ile

\begin{tabular}{|c|c|c|}
\hline $\begin{array}{l}\text { doubletime } \\
\text { gene, } \\
\text { Drosophila }\end{array}$ & $d b t^{P}$ & $\begin{array}{l}\text { P-element insertion } \\
\text { in intron } 2 \text { disrupts } \\
\text { gene function }\end{array}$ \\
\hline $\begin{array}{l}\text { doubletime } \\
\text { gene, } \\
\text { Drosophila }\end{array}$ & $d b t^{a r}$ & $\begin{array}{l}\text { single-nucleotide } \\
\text { exchange leads } \\
\text { to His-126 } \rightarrow \mathrm{Tyr}\end{array}$ \\
\hline $\begin{array}{l}\text { period } 2 \\
\text { gene, } \\
\text { human }\end{array}$ & $\begin{array}{c}\text { Per2S662G } \\
\text { (FASPS) }\end{array}$ & $\begin{array}{l}\text { single-nucleotide } \\
\text { exchange leads } \\
\text { to Ser- } 662 \rightarrow \text { Gly }\end{array}$ \\
\hline 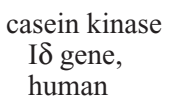 & $\begin{array}{r}C K I \delta T 44 A \\
(\text { FASPS) }\end{array}$ & $\begin{array}{l}\text { single-nucleotide } \\
\text { exchange leads } \\
\text { to Thr-44 } \rightarrow \text { Ala }\end{array}$ \\
\hline $\begin{array}{l}\text { casein kinase } \\
\text { I } \varepsilon \text { gene, } \\
\text { hamster }\end{array}$ & $\begin{array}{l}\text { CKIER } 178 C \\
\quad(\text { tau })\end{array}$ & $\begin{array}{l}\text { single-nucleotide } \\
\text { exchange leads } \\
\text { to Arg-178 } \rightarrow \text { Cys }\end{array}$ \\
\hline
\end{tabular}

long-period rhythms $(27 \mathrm{hr})$ in eclosion and behavior

heterozygous: normal behavioral rhythms homozygous: lethal

homozygous: arrhythmic behavior

FASPS, 4-hr advance of circadian parameters, short endogenous period

FASPS, 4-hr advance of circadian parameters, short endogenous period

tau phenotype: short-period behavioral rhythms heterozygous: $22 \mathrm{hr}$ heterozygous: $20 \mathrm{hr}$ accelerated PER accumulation in the cytoplasm and disappearance from the nuclei; reduced kinase activity in vitro

accumulation pattern of PER is delayed; longer persistence in the declining phase of the circadian cycle; reduced kinase activity in vitro

hypophosphorylated PER accumulates to high levels in homozygous $d b t^{P}$ larvae

hypo- and hyperphosphorylated PER accumulates to high levels; PER oscillation is stopped

hypophosphorylation of PER2 protein in vitro; reduced PER2 stability due to decreased nuclear retention

reduced kinase activity in vitro

reduced kinase activity in vitro, premature nuclear clearance of PER 1 and PER2 in the SCN; increased phosphorylation of PER proteins in cells
Kloss et al. (1998);

Price et al. (1998);

Bao et al. (2001);

Preuss et al.

(2004)

Kloss et al. (1998);

Price et al. (1998); Suri et al. (2000);

Preuss et al. (2004)

Kloss et al. (1998);

Price et al. (1998)

Rothenfluh et al. (2000)

Toh et al. (2001); Vanselow et al. (2006)

Xu et al. (2005)

Ralph and Menaker (1988); Lowrey et al. (2000); Dey et al. (2005); Gallego et al. (2006b) 


\section{PHOSPHORYLATION IN THE MAMMALIAN CIRCADIAN CLOCK}

results in an arginine-to-cysteine substitution at amino acid 178 of CKIE. In vitro, recombinantly produced tau kinase has a reduced activity, whereas the binding to mPER1 and mPER2 is not impaired (Lowrey et al. 2000). This findings suggest a possible reduction in the phosphorylation of the PER proteins by CKIe(tau) also in vivo. Unexpectedly, however, tau mutant hamsters exhibit robust circadian rhythms in both PER protein abundance and PER phosphorylation without substantial differences in the degree of hyperphosphorylation, although the overall amount of PER protein is reduced (Lee et al. 2001). Interestingly, an accelerated nuclear clearance of PER proteins in the SCN of tau mutant hamsters has been reported (Dey et al. 2005).

A second phosphorylation-based circadian phenotype is the FASPS phenotype in humans. The heredity transmission of FASPS follows an autosomal dominant pattern (Jones et al. 1999; Toh et al. 2001; Xu et al. 2005). Affected individuals are extreme morning larks with an approximately 4-5-hour advance of sleep onset and offset, temperature, and hormonal rhythms. The endogenous period of one affected individual was measured to be 23.3 hours (Jones et al. 1999), which is about 1 hour shorter than the average population (Czeisler et al. 1999). Up to now, genetic analyses have identified two singlenucleotide exchanges in human clock genes correlating with the FASPS phenotype. In one case, the human Per2 gene is mutated, which leads to a serine-to-glycine exchange at residue 662 of hPER2 (Toh et al. 2001). This region of PER2 is highly conserved within all paralog PER proteins in mammals. Notably, the serine residue mutated in FASPS is the first residue in a cluster of serine and threonine residues forming a canonical CKI $/ \delta$ phosphorylation/recognition motif $\left(\mathrm{pS} / \mathrm{pT} / \mathrm{pYX} \mathrm{X}_{(1-2)} \mathrm{S} / \mathrm{T} ; \mathrm{X}=\right.$ any amino acid) (Flotow et al. 1990). In vitro, the FASPS mutation causes hypophosphorylation of PER2 when phosphorylated with CKIE (Toh et al. 2001).

In a second form of FASPS, the human $C K I \delta$ gene is mutated. Here, a threonine-to-alanine substitution at amino acid 44 in the CKI $\delta$ protein has been identified. As in the case of tau, recombinantly produced CKI $\delta$-T44A has reduced kinase activity in vitro. Interestingly, transgenic mice containing a human bacterial artificial chromosome (BAC) clone expressing the CKIס-T44A mutant show a shortened circadian period, whereas the expression of hCKI $\delta$-T44A in flies leads to a period lengthening (Xu et al. 2005), although the respective position is highly conserved between mammalian CKI $\delta$ and DBT. This suggests that reducing the activity of CKI $\delta$ can result in opposite period phenotypes depending on the cellular context and/or the substrates.

Until recently, the molecular mechanisms of both the tau and the FASPS phenotype were postulated as follows: In tau, the reduced kinase activity and in FASPS, the lack of a specific phosphorylatable residue in PER2 both result in hypophosphorylation of PER proteins. Because phosphorylation triggers degradation, it has been assumed that PER hypophosphorylation would lead to a stabilization of PER proteins and thus to a more rapid PER accumulation in the cytoplasm, followed by an advanced nuclear localization of PER proteins. An earlier repression of CLOCK-
BMAL1 transcriptional activity then would lead to shorter circadian periods (Lowrey et al. 2000; Dey et al. 2005). However, we propose an alternative mechanism (see below). Although the available data strongly suggest a disturbance of PER phosphorylation as the molecular basis for tau and FASPS, direct evidence for altered phosphorylation patterns in vivo was still missing.

\section{Mapping of Phosphorylation Sites in Clock Proteins}

The examples described above underline the enormous importance of proper phosphorylation of PER proteins and possibly other clock components for the generation and dynamics of circadian oscillations. The circadian phasedependent gradual decrease of PER's electrophoretic mobility indicates that several distinguishable PER phosphorylation species exist. In addition, the differential effects of some mutations on PER stability and subcellular localization argue for specific phosphorylation sites influencing these properties. To elucidate how phosphorylation at particular sites regulates the temporal and spatial properties of PER proteins, a detailed map of endogenous PER phosphorylation sites is needed. Knowing these sites would allow for the study of their specific function and may provide hints toward the identification of the kinases responsible for phosphorylation. Previous studies analyzed single phosphorylation sites in mPER1 with respect to their functional roles (Vielhaber et al. 2000; Takano et al. 2004). Until recently, however, no systematic effort to comprehensively map in vivo clock protein phosphorylation sites has been undertaken.

We have established a novel mass spectrometric technique that allows sensitive and comprehensive mapping of protein phosphorylation sites. We use this technique to analyze phosphorylation sites in clock proteins in a systematic manner. Immunoprecipitated proteins are subjected to in-gel digests with a set of four different proteases. Phosphopeptides are enriched on titanesphere columns and then analyzed by ultra-low-flow nanoLCMS/MS (Schlosser et al. 2005).

The first clock protein that we analyzed was mouse PER2, because it is the best-characterized circadian phosphoprotein to date with a very pronounced temporal phosphorylation profile. For that purpose, we generated a HEK 293 cell line stably expressing carboxy-terminal V5tagged mPER2 for efficient immunoprecipitation. The mass spectrometric approach mentioned above led to the identification of 21 (of 275 theoretically possible) endogenous phosphorylation sites in mPER2 (exclusively serine and threonine residues). Interestingly, we found the serine residue phosphorylated, which is mutated in FASPS (Vanselow et al. 2006). This is the first direct evidence that the FASPS site is indeed a target for phosphorylation in living cells. Generally, many of the detected phosphorylation sites are located close to the previously identified $\mathrm{CKI} / \delta$ - $\delta$-binding domain (amino acids 555-754) (Akashi et al. 2002) or surrounding the nuclear export sequence-2 (NES-2 amino acids 983-990) (Yagita et al. 2002). Further phosphorylation sites are concentrated at the carboxyl and amino termini, and no phosphorylation site was found in the PAS domain region. 
To investigate the function of these 21 phosphorylation sites of mPER2, we established a reductionist model system, which is based on the expression of phosphositemutated mPER2 proteins in synchronized fibroblasts. Potential alterations in the oscillation properties are analyzed using luciferase-based circadian reporters. Fibroblast cell lines like Rat-1 and NIH-3T3 have been demonstrated to show self-sustained circadian oscillations with properties very similar to those of the SCNintrinsic cellular oscillator (Balsalobre et al. 1998; Yagita et al. 2001; Brown et al. 2005b). In the first step, we generated an NIH-3T3 Flp-In host cell line harboring the Flp recombinase target (FRT) site (O'Gorman et al. 1991). This ensures the expression of different mPER2 variants from the same chromosomal location in the fibroblast genome. In a second step, expression cell lines of PER2 variants were generated by recombining mutant constructs into the FRT site of the NIH-3T3 genome. Thus, differences in the oscillation dynamics of the resulting expression cell lines or in the biochemical properties of the PER2 variants are directly caused by the PER 2 protein mutations, and artifacts due to chromosomal positioning effects or variation of integration events were excluded.

We were particularly interested in investigating the functional role of the PER2 phosphorylation at the FASPS region, because this region has been implicated to be crucial for the development of FASPS. To this end, we generated different variants of mPER2 affecting the FASPS region (FASPS [S659G], mut-7 [S659G, S662A, S665A, S668A, S670A, S671A, TS672A], and S659D) by site-directed mutagenesis. These PER2 variants were then expressed in our NIH-3T3 Flp-In cell line. The circadian oscillations of the resulting cell lines were recorded continuously in a luminometer. Interestingly, the oscillation dynamics of PER2-FASPS expressing cells is very similar to the behavior of FASPS patients: an early phase of entrainment and a short free-running period (Fig. 1) (Vanselow et al. 2006).

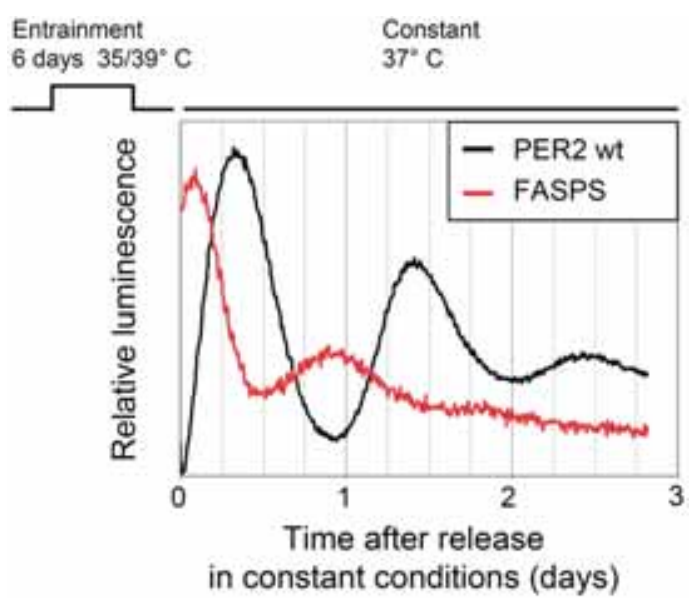

Figure 1. Expression of PER2-FASPS causes an advanced phase and a shorter period in oscillating NIH-3T3 fibroblasts after a several day temperature entrainment regime. Real-time circadian oscillations of luciferase reporter activity were recorded in a luminometer. (Reprinted, with permission, from Vanselow et al. 2006.)

\section{Functional Different Phosphorylation Sites in mPER2: A Molecular Basis for FASPS and tau}

We next wanted to elucidate the molecular processes that are altered in FASPS. In principle, there are several possibilities of how the phosphorylation at the FASPS position and presumably also the following serine and threonine residues of the CKI $\varepsilon / \delta$ phosphorylation cluster might influence the oscillation dynamics. These include the regulation of PER2 stability, PER2 subcellular localization, and/or PER2 inhibition of CLOCK-BMAL1 activity.

We have analyzed all of these possibilities. Alterations in the ability of PER2-FASPS to inhibit trans-activation by CLOCK:BMAL1 were not observed. Surprisingly, however, we found that the stability of PER2-FASPS is not - as it might be expected-increased compared to PER2 wild-type protein but is substantially reduced. Although the protein half-life of PER2-wt is about 3 hours, the half-life of PER2-FASPS is reduced to about 1.5 hours. The destabilization is even more pronounced in the PER2-mut-7 variant, in which the whole CKI $\varepsilon / \delta$ phosphorylation cluster is mutated, whereas the stability of the PER2-S659D mutant is not altered (Fig. 2) (Vanselow et al. 2006). Coexpression studies with CKIE revealed that the decreased stability of PER2-FASPS is due to its higher sensitivity toward CKIE-mediated proteasomal degradation. Notably, Takano and colleagues made similar observations when analyzing the corresponding sites in PER1. Coexpression of the PER1 mutant with CKIE resulted in a reduced stability and higher phosphorylation state (Takano et al. 2004; Takano and Nagai 2006). Thus, phosphorylation of PER proteins does not always trigger the degradation pathway but can have differential functions. Although phosphorylation in the FASPS region stabilizes PER proteins, phosphorylation at other residues promotes proteasomal degradation.

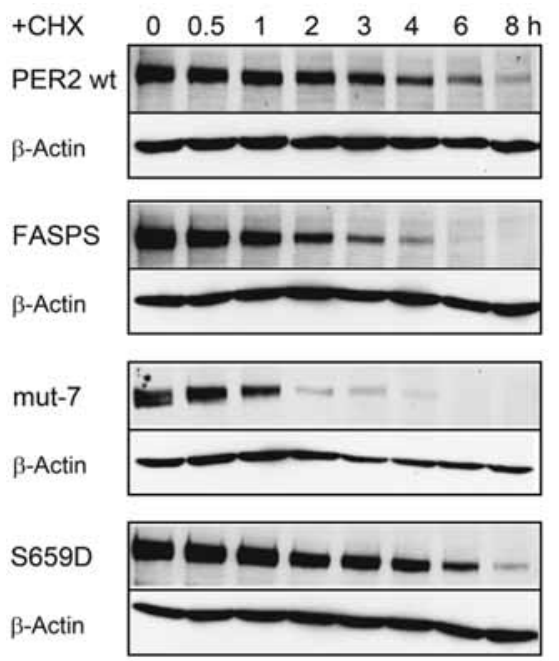

Figure 2. The FASPS mutation destabilizes the PER2 protein. NIH-3T3 cells stably expressing indicated PER2 variants were treated for up to 8 hours with the protein translation inhibitor cycloheximid (CHX). The PER2 protein amount was determined by SDS-PAGE/western blotting. (Reprinted, with permission, from Vanselow et al. 2006.) 


\section{PHOSPHORYLATION IN THE MAMMALIAN CIRCADIAN CLOCK}

Next, we wanted to answer the question of why phosphorylation in the FASPS region stabilizes PER2. To this end, we tested whether stabilization is an indirect consequence of an altered subcellular localization of PER2. Although we could not detect a different nuclear import rate of PER2-FASPS, we did observe an accelerated nuclear clearance of the PER2-FASPS protein (Fig. 3)

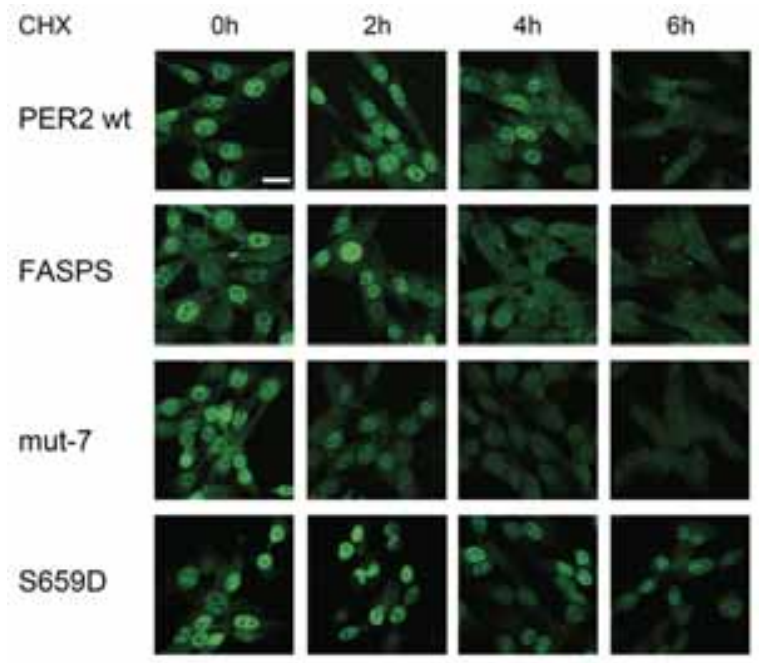

Figure 3. The FASPS mutation leads to a premature nuclear clearance. PER2 variant expressing NIH-3T3 cells were treated with the protein translation inhibitor cycloheximid (CHX) for the indicated times. Subcellular localization of PER2 proteins was visualized after immunostaining by confocal fluorescent microscopy. (Reprinted, with permission, from Vanselow et al. 2006.)
(Vanselow et al. 2006). This result is similar to observations for PER1 and PER2 in the SCN of tau mutant hamsters (Dey et al. 2005). Thus, phosphorylation at the FASPS region increases nuclear retention of PER2, thereby protecting it from cytoplasmic degradation by the proteasome. In the FASPS mutation, however, we propose that the mutant PER2 protein is prematurely exported, which leads to an earlier release of CLOCKBMAL1 repression and earlier restart of a new cycle (Fig. 4) (Vanselow et al. 2006).

Xu et al. (2007) generated transgenic mice to study the molecular mechanisms underlying FASPS. The protein levels of PER2-FASPS (especially in the nuclear fraction) in the liver of those transgenic mice as well as in fibroblasts from skin biopsies of FASPS patients were found to be reduced as compared to controls. As in our study, this indicates a decreased stability of the PER2-FASPS protein. However, because the authors did not find a stabilizing effect of the proteasomal inhibitor MG132, they excluded the possibility of an altered stability of PER2FASPS as the primary consequence of the mutation. Instead, they found a reduction of the Per 2 mRNA in FASPS and concluded that phosphorylation at the FASPS position has an impact on Per2 transcription, a result we did not observe in our model system.

Functionally different phosphorylation sites in PER2 may also - at least qualitatively - explain the molecular mechanism for the $C K I \delta$-based variant of FASPS as well as the tau phenotype in the hamster. In contrast to the Per2-based version of FASPS, CKI $\delta$-based FASPS and tau could be explained by an altered ability of the kinases to phosphorylate PER2 (note, the reduced kinase activity

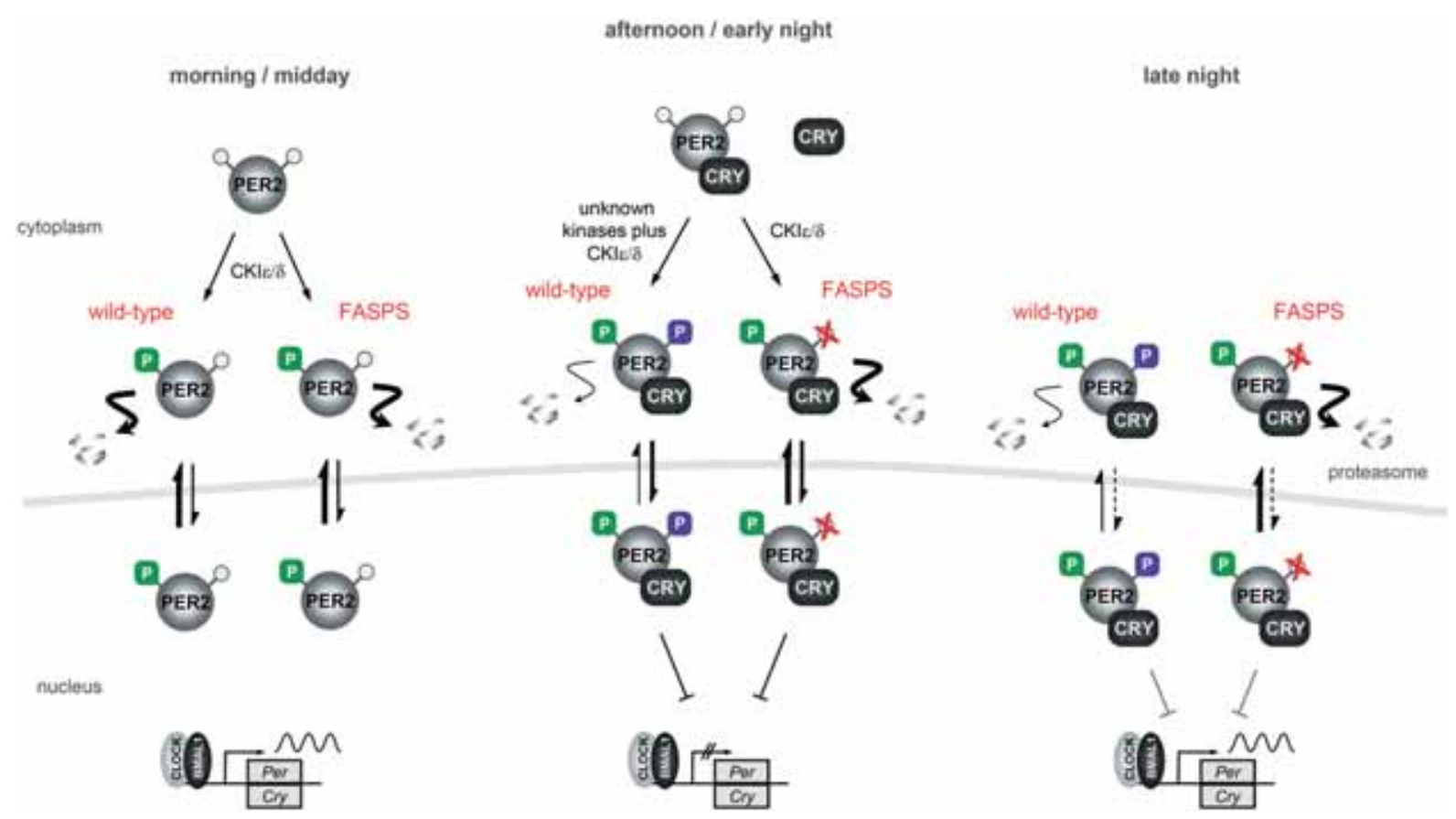

Figure 4. Functional different phosphorylation sites in PER2 as the molecular basis for FASPS. The PER2 protein contains at least two functionally different phosphorylation sites - one kind primarily mediating proteasomal degradation (green), and the other enhancing nuclear retention (purple). For mechanistic details, see the main text. (Reprinted, with permission, from Vanselow et al. 2006.) 
of CKI $\delta$-T44A and CKIE[tau] in vitro). Here, the key assumption is that the overall decrease in kinase activity does not lead to an evenly reduction of phosphorylation at all PER2 phosphorylation sites but to a specific decrease or inability to phosphorylate specific residues in the protein. According to our model, both CKI $\delta$-T44A and $\mathrm{CKI}($ tau $)$ are impaired in the phosphorylation of sites, which stabilize PER2 (maybe even at the FASPS region), whereas the phosphorylation at destabilizing degradationpromoting sites is unaffected or less affected. This may lead to a premature nuclear clearance of PER proteins and therefore to the observed early phase and short period of the FASPS individuals or tau hamsters, respectively.

To conceptualize our results, and to better understand their implications for circadian rhythm generation in a more quantitative manner, we constructed a mathematical model of PER2 phosphorylation in the circadian system (Vanselow et al. 2006). The model shows robust self-sustained oscillations with a period of 24 hours and delay of 6 hours between the Per 2 mRNA and PER2 protein rhythms. Furthermore, the simulation of an intervention with phosphorylation events that promote nuclear retention produces a short period and early phase as observed for FASPS patients. The model was also successfully applied to deduce testable predictions for the effects of specific system perturbations. For example, one biochemical prediction from the mathematical model is that the coexpression of PER2-wt with CKIE(tau) or CKI $\delta$-T44A should destabilize the PER2-wt protein to a higher degree than the coexpression of PER2-wt with CKIr(wt). Concomitantly, the destabilizing effects of CKI $\varepsilon, \mathrm{CKI} \varepsilon($ tau $)$, and CKI $\delta$-T44A on PER2-FASPS should be similar to each other. We tested these predictions for the tau kinase in cultured cells, and as predicted, the destabilizing effect of CKIE(tau) on PER2wt was much more pronounced than that of the wild-type kinase. Furthermore, the destabilizing effect of CKIE(tau) on PER2-wt and PER2-FASPS was comparable.

In line with these results, Gallego et al. (2006b) found a higher destabilizing effect of the tau kinase on PER1 and PER2 in cell culture. The starting point of their experiments was mathematical modeling, which claimed an increased kinase activity (gain-of-function) for CKIE(tau) in vivo as the only possible explanation for the shortperiod phenotype. With respect to the tau mutation, our model and that of Gallego et al. (2006b) offer alternative but not mutually exclusive explanations. We favor a mechanism where a reduced kinase activity of mutant CKI $\varepsilon$ leads to an altered, less nuclear localization of PER proteins (as observed by Dey et al. 2005). Because phosphorylation-induced proteasomal degradation of PER proteins is primarily a cytosolic event (Vanselow et al. 2006), the overall phosphorylation of the PER proteins in tau hamsters may even increase (as observed by Gallego et al. 2006b). We propose that this increased phosphorylation is primarily due to a higher amount of PER substrate in the cytoplasm, rather than to an increased kinase activity. Nevertheless, the available data on the tau mutation fit both models. With respect to the Per2-based FASPS mutation, however, the cellular localization data (Vanselow et al. 2006) strongly favor the localizationdependent model that we postulate.

\section{CONCLUSIONS}

Experimental results from the last two decades underline the crucial importance of posttranslational mechanisms for both the generation and dynamics of circadian rhythms. Specifically, the reversible phosphorylation of the PER proteins in Drosophila and mammals seems to have a key role for the correct timing of nuclear repression. Animals with altered PER phosphorylation (by either a mutant PER protein or a mutant kinase) have striking circadian phenotypes at the behavioral as well as the molecular level. Biochemical analyses indicate that phosphorylation regulates not only the stability of PER proteins, but also their subcellular localization and inhibitory activity. Until recently, it was unknown which of the many potential phosphorylation sites of PER proteins are functionally relevant in vivo. Our aim is to perform a comprehensive phosphorylation function analysis of clock proteins. For that purpose, we developed an optimized mass spectrometric approach and were able to identify endogenous phosphorylation sites of mPER2. In a cell culture model system, we then studied the oscillation dynamics of cells expressing phosphosite-mutant PER proteins. This approach allowed us to analyze the molecular basis for the human FASPS. Remarkably, the phosphorylation site in PER2, which is mutated in FASPS, stabilizes the protein by means of nuclear retention and thus protects it from cytosolic degradation. On the basis of our data, we proposed a model of functionally different phosphorylation sites in PER2, which is also able to explain additional phosphorylationbased circadian phenotypes. We are currently investigating the role of other identified phosphorylation sites, especially with respect to potential destabilizing effects.

\section{ACKNOWLEDGMENTS}

We thank Ute Abraham for critical reading of the manuscript. Research in our lab is supported by the Deutsche Forschungsgemeinschaft (grants SFB 618, SFB 740 ) and the Sixth EU framework programme EUCLOCK.

\section{REFERENCES}

Akashi M., Tsuchiya Y., Yoshino T., and Nishida E. 2002. Control of intracellular dynamics of mammalian period proteins by casein kinase I epsilon (CKIepsilon) and CKIdelta in cultured cells. Mol. Cell. Biol. 22: 1693.

Akten B., Jauch E., Genova G.K., Kim E.Y., Edery I., Raabe T., and Jackson F.R. 2003. A role for CK2 in the Drosophila circadian oscillator. Nat. Neurosci. 6: 251.

Balsalobre A., Damiola F., and Schibler U. 1998. A serum shock induces circadian gene expression in mammalian tissue culture cells. Cell 93: 929.

Bao S., Rihel J., Bjes E., Fan J.Y., and Price J.L. 2001. The Drosophila double-timeS mutation delays the nuclear accumulation of period protein and affects the feedback regulation of period mRNA. J. Neurosci. 21: 7117.

Barnes J.W., Tischkau S.A., Barnes J.A., Mitchell J.W., Burgoon P.W., Hickok J.R., and Gillette M.U. 2003. Requirement of mammalian Timeless for circadian rhythmicity. Science 302: 439.

Brown S.A., Ripperger J., Kadener S., Fleury-Olela F., Vilbois F., Rosbash M., and Schibler U. 2005a. PERIOD1-associated proteins modulate the negative limb of the mammalian circadian oscillator. Science 308: 693. 


\section{PHOSPHORYLATION IN THE MAMMALIAN CIRCADIAN CLOCK}

Brown S.A., Fleury-Olela F., Nagoshi E., Hauser C., Juge C., Meier C.A., Chicheportiche R., Dayer J.M., Albrecht U., and Schibler U. 2005b. The period length of fibroblast circadian gene expression varies widely among human individuals. PLoS Biol. 3: e338.

Busino L., Bassermann F., Maiolica A., Lee C., Nolan P.M., Godinho S.I., Draetta G.F., and Pagano M. 2007. SCFFbxl3 controls the oscillation of the circadian clock by directing the degradation of cryptochrome proteins. Science 316: 900.

Camacho F., Cilio M., Guo Y., Virshup D.M., Patel K., Khorkova O., Styren S., Morse B., Yao Z., and Keesler G.A. 2001. Human casein kinase Idelta phosphorylation of human circadian clock proteins period 1 and 2. FEBS Lett. 489: 159.

Cardone L., Hirayama J., Giordano F., Tamaru T., Palvimo J.J., and Sassone-Corsi P. 2005. Circadian clock control by SUMOylation of BMAL1. Science 309: 1390

Curtin K.D., Huang Z.J., and Rosbash M. 1995. Temporally regulated nuclear entry of the Drosophila period protein contributes to the circadian clock. Neuron 14: 365.

Cyran S.A., Yiannoulos G., Buchsbaum A.M., Saez L., Young M.W., and Blau J. 2005. The double-time protein kinase regulates the subcellular localization of the Drosophila clock protein period. J. Neurosci. 25: 5430.

Czeisler C.A., Duffy J.F., Shanahan T.L., Brown E.N., Mitchell J.F., Rimmer D.W., Ronda J.M., Silva E.J., Allan J.S., Emens J.S., Dijk D.J., and Kronauer R.E. 1999. Stability, precision, and near-24-hour period of the human circadian pacemaker. Science 284: 2177

Dey J., Carr A.J., Cagampang F.R., Semikhodskii A.S., Loudon A.S., Hastings M.H., and Maywood E.S. 2005. The tau mutation in the Syrian hamster differentially reprograms the circadian clock in the SCN and peripheral tissues. J. Biol. Rhythms 20: 99.

Edery I., Zwiebel L.J., Dembinska M.E., and Rosbash M. 1994. Temporal phosphorylation of the Drosophila period protein. Proc. Natl. Acad. Sci. 91: 2260.

Eide E.J., Vielhaber E.L., Hinz W.A., and Virshup D.M. 2002. The circadian regulatory proteins BMAL1 and cryptochromes are substrates of casein kinase Iepsilon. J. Biol. Chem. 277: 17248.

Eide E.J., Woolf M.F., Kang H., Woolf P., Hurst W., Camacho F., Vielhaber E.L., Giovanni A., and Virshup D.M. 2005. Control of mammalian circadian rhythm by CKIepsilon-regulated proteasome-mediated PER2 degradation. Mol. Cell Biol. 25: 2795 .

Flotow H., Graves P.R., Wang A.Q., Fiol C.J., Roeske R.W., and Roach P.J. 1990. Phosphate groups as substrate determinants for casein kinase I action. J. Biol. Chem. 265: 14264.

Fujimoto Y., Yagita K., and Okamura H. 2006. Does mPER2 protein oscillate without its coding mRNA cycling?: Posttranscriptional regulation by cell clock. Genes Cells 11: 525 .

Gallego M., Kang H., and Virshup D.M. 2006a. Protein phosphatase 1 regulates the stability of the circadian protein PER2. Biochem. J. 399: 169.

Gallego M., Eide E.J., Woolf M.F., Virshup D.M., and Forger D.B. 2006b. An opposite role for tau in circadian rhythms revealed by mathematical modeling. Proc. Natl. Acad. Sci. 103: 10618

Godinho S.I., Maywood E.S., Shaw L., Tucci V., Barnard A.R., Busino L., Pagano M., Kendall R., Quwailid M.M., Romero M.R., O'Neill J., Chesham J.E., Brooker D., Lalanne Z., Hastings M.H., and Nolan P.M. 2007. The after-hours mutant reveals a role for Fbxl3 in determining mammalian circadian period. Science 316: 897

Grima B., Lamouroux A., Chelot E., Papin C., LimbourgBouchon B., and Rouyer F. 2002. The F-box protein slimb controls the levels of clock proteins period and timeless. Nature 420: 178

Harada Y., Sakai M., Kurabayashi N., Hirota T., and Fukada Y. 2005. Ser-557-phosphorylated mCRY2 is degraded upon synergistic phosphorylation by glycogen synthase kinase- 3 beta. J. Biol. Chem. 280: 31714 .

Hardin P.E., Hall J.C., and Rosbash M. 1990. Feedback of the Drosophila period gene product on circadian cycling of its messenger RNA levels. Nature 343: 536.
Hofmann K.P., Spahn C.M., Heinrich R., and Heinemann U. 2006. Building functional modules from molecular interactions. Trends Biochem. Sci. 31: 497.

Holmberg C.I., Tran S.E., Eriksson J.E., and Sistonen L. 2002. Multisite phosphorylation provides sophisticated regulation of transcription factors. Trends Biochem. Sci. 27: 619 .

Honma S., Kawamoto T., Takagi Y., Fujimoto K., Sato F., Noshiro M., Kato Y., and Honma K. 2002. Dec1 and Dec2 are regulators of the mammalian molecular clock. Nature 419: 841

Iitaka C., Miyazaki K., Akaike T., and Ishida N. 2005. A role for glycogen synthase kinase-3beta in the mammalian circadian clock. J. Biol. Chem. 280: 29397.

Jones C.R., Campbell S.S., Zone S.E., Cooper F., DeSano A., Murphy P.J., Jones B., Czajkowski L., and Ptacek L.J. 1999. Familial advanced sleep-phase syndrome: A short-period circadian rhythm variant in humans. Nat. Med. 5: 1062.

Keesler G.A., Camacho F., Guo Y., Virshup D., Mondadori C., and Yao Z. 2000. Phosphorylation and destabilization of human period I clock protein by human casein kinase I epsilon. Neuroreport 11: 951

Kim E.Y. and Edery I. 2006. Balance between DBT/CKIepsilon kinase and protein phosphatase activities regulate phosphorylation and stability of Drosophila CLOCK protein. Proc. Natl. Acad. Sci. 103: 6178.

Kloss B., Price J.L., Saez L., Blau J., Rothenfluh A., Wesley C.S., and Young M.W. 1998. The Drosophila clock gene double-time encodes a protein closely related to human casein kinase Iepsilon. Cell 94: 97.

Ko H.W., Jiang J., and Edery I. 2002. Role for Slimb in the degradation of Drosophila Period protein phosphorylated by Doubletime. Nature 420: 673.

Konopka R.J. and Benzer S. 1971. Clock mutants of Drosophila melanogaster. Proc. Natl. Acad. Sci. 68: 2112.

Lee C., Etchegaray J.P., Cagampang F.R., Loudon A.S., and Reppert S.M. 2001. Posttranslational mechanisms regulate the mammalian circadian clock. Cell 107: 855.

Lin J.M., Schroeder A., and Allada R. 2005. In vivo circadian function of casein kinase 2 phosphorylation sites in Drosophila PERIOD. J. Neurosci. 25: 11175.

Lin J.M., Kilman V.L., Keegan K., Paddock B., Emery-Le M., Rosbash M., and Allada R. 2002. A role for casein kinase 2alpha in the Drosophila circadian clock. Nature 420: 816.

Lowrey P.L., Shimomura K., Antoch M.P., Yamazaki S., Zemenides P.D., Ralph M.R., Menaker M., and Takahashi J.S. 2000. Positional syntenic cloning and functional characterization of the mammalian circadian mutation tau. Science 288: 483 .

Martinek S., Inonog S., Manoukian A.S., and Young M.W. 2001. A role for the segment polarity gene shaggy/GSK-3 in the Drosophila circadian clock. Cell 105: 769.

Nakajima M., Imai K., Ito H., Nishiwaki T., Murayama Y., Iwasaki H., Oyama T., and Kondo T. 2005. Reconstitution of circadian oscillation of cyanobacterial KaiC phosphorylation in vitro. Science 308: 414.

Nawathean P. and Rosbash M. 2004. The doubletime and CKII kinases collaborate to potentiate Drosophila PER transcriptional repressor activity. Mol. Cell 13: 213.

Nishii K., Yamanaka I., Yasuda M., Kiyohara Y.B., Kitayama Y., Kondo T., and Yagita K. 2006. Rhythmic post-transcriptional regulation of the circadian clock protein mPER2 in mammalian cells: A real-time analysis. Neurosci. Lett. 401: 44.

O'Gorman S., Fox D.T., and Wahl G.M. 1991. Recombinasemediated gene activation and site-specific integration in mammalian cells. Science 251: 1351.

Partch C.L., Shields K.F., Thompson C.L., Selby C.P., and Sancar A. 2006. Posttranslational regulation of the mammalian circadian clock by cryptochrome and protein phosphatase 5. Proc. Natl. Acad. Sci. 103: 10467.

Preitner N., Damiola F., Lopez-Molina L., Zakany J., Duboule D., Albrecht U., and Schibler U. 2002. The orphan nuclear receptor REV-ERBalpha controls circadian transcription within the positive limb of the mammalian circadian oscillator. Cell 110: 251 . 
Preuss F., Fan J.Y., Kalive M., Bao S., Schuenemann E., Bjes E.S., and Price J.L. 2004. Drosophila doubletime mutations which either shorten or lengthen the period of circadian rhythms decrease the protein kinase activity of casein kinase I. Mol. Cell. Biol. 24: 886.

Price J.L., Blau J., Rothenfluh A., Abodeely M., Kloss B., and Young M.W. 1998. double-time is a novel Drosophila clock gene that regulates PERIOD protein accumulation. Cell 94: 83 .

Ralph M.R. and Menaker M. 1988. A mutation of the circadian system in golden hamsters. Science 241: 1225.

Reischl S., Vanselow K., Westermark P.O., Thierfelder N., Maier B., Herzel H., and Kramer A. 2007. B-TrCP1-mediated degradation of PERIOD2 is essential for circadian dynamics. J. Biol. Rhythms 22: 375.

Rothenfluh A., Abodeely M., and Young M.W. 2000. Shortperiod mutations of per affect a double-time-dependent step in the Drosophila circadian clock. Curr. Biol. 10: 1399.

Sangoram A.M., Saez L., Antoch M.P., Gekakis N., Staknis D., Whiteley A., Fruechte E.M., Vitaterna M.H., Shimomura K., King D.P., Young M.W., Weitz C.J., and Takahashi J.S. 1998. Mammalian circadian autoregulatory loop: A timeless ortholog and mPer1 interact and negatively regulate CLOCKBMAL1-induced transcription. Neuron 21: 1101.

Sathyanarayanan S., Zheng X., Xiao R., and Sehgal A. 2004. Posttranslational regulation of Drosophila PERIOD protein by protein phosphatase 2A. Cell 116: 603 .

Schlosser A., Vanselow J.T., and Kramer A. 2005. Mapping of phosphorylation sites by a multi-protease approach with specific phosphopeptide enrichment and NanoLC-MS/MS analysis. Anal. Chem. 77: 5243.

Shirogane T., Jin J., Ang X.L., and Harper J.W. 2005. SCFbetaTRCP controls clock-dependent transcription via casein kinase 1-dependent degradation of the mammalian period-1 (Per1) protein. J. Biol. Chem. 280: 26863.

Siepka S.M., Yoo S.H., Park J., Song W., Kumar V., Hu Y., Lee C., and Takahashi J.S. 2007. Circadian mutant Overtime reveals F-box protein FBXL3 regulation of cryptochrome and period gene expression. Cell 129: 1011

Sugano S., Andronis C., Ong M.S., Green R.M., and Tobin E.M. 1999. The protein kinase CK2 is involved in regulation of circadian rhythms in Arabidopsis. Proc. Natl. Acad. Sci. 96: 12362.

Suri V., Hall J.C., and Rosbash M. 2000. Two novel doubletime mutants alter circadian properties and eliminate the delay between RNA and protein in Drosophila. J. Neurosci. 20: 7547.

Takano A. and Nagai K. 2006. Serine 714 might be implicated in the regulation of the phosphorylation in other areas of mPer1 protein. Biochem. Biophys. Res. Commun. 346: 95.

Takano A., Isojima Y., and Nagai K. 2004. Identification of mPer1 phosphorylation sites responsible for the nuclear entry. J. Biol. Chem. 279: 32578.

Takano A., Shimizu K., Kani S., Buijs R.M., Okada M., and Nagai K. 2000. Cloning and characterization of rat casein kinase 1epsilon. FEBS Lett. 477: 106.
Toh K.L., Jones C.R., He Y., Eide E.J., Hinz W.A., Virshup D.M., Ptacek L.J., and Fu Y.H. 2001. An hPer2 phosphorylation site mutation in familial advanced sleep phase syndrome. Science 291: 1040.

Tomita J., Nakajima M., Kondo T., and Iwasaki H. 2005. No transcription-translation feedback in circadian rhythm of KaiC phosphorylation. Science 307: 251

Vanselow K., Vanselow J.T., Westermark P.O., Reischl S., Maier B., Korte T., Herrmann A., Herzel H., Schlosser A., and Kramer A. 2006. Differential effects of PER2 phosphorylation: Molecular basis for the human familial advanced sleep phase syndrome (FASPS). Genes Dev. 20: 2660.

Vielhaber E., Eide E., Rivers A., Gao Z.H., and Virshup D.M. 2000. Nuclear entry of the circadian regulator mPER1 is controlled by mammalian casein kinase I epsilon. Mol. Cell. Biol. 20: 4888.

Xu Y., Toh K.L., Jones C.R., Shin J.Y., Fu Y.H., and Ptacek L.J. 2007. Modeling of a human circadian mutation yields insights into clock regulation by PER2. Cell 128: 59 .

Xu Y., Padiath Q.S., Shapiro R.E., Jones C.R., Wu S.C., Saigoh N., Saigoh K., Ptacek L.J., and Fu Y.H. 2005. Functional consequences of a CKIdelta mutation causing familial advanced sleep phase syndrome. Nature 434: 640.

Yagita K., Tamanini F., Der Horst G.T., and Okamura H. 2001. Molecular mechanisms of the biological clock in cultured fibroblasts. Science 292: 278.

Yagita K., Tamanini F., Yasuda M., Hoeijmakers J.H., van der Horst G.T., and Okamura H. 2002. Nucleocytoplasmic shuttling and mCRY-dependent inhibition of ubiquitylation of the MPER2 clock protein. EMBO J. 21: 1301.

Yang Y., Cheng P., and Liu Y. 2002. Regulation of the Neurospora circadian clock by casein kinase II. Genes Dev. 16: 994.

Yang Z. and Sehgal A. 2001. Role of molecular oscillations in generating behavioral rhythms in Drosophila. Neuron 29: 453.

Yin L., Wang J., Klein P.S., and Lazar M.A. 2006. Nuclear receptor Rev-erbalpha is a critical lithium-sensitive component of the circadian clock. Science 311: 1002.

Yu Q., Jacquier A.C., Citri Y., Hamblen M., Hall J.C., and Rosbash M. 1987. Molecular mapping of point mutations in the period gene that stop or speed up biological clocks in Drosophila melanogaster. Proc. Natl. Acad. Sci. 84: 784.

Yu W., Zheng H., Houl J.H., Dauwalder B., and Hardin P.E. 2006. PER-dependent rhythms in CLK phosphorylation and E-box binding regulate circadian transcription. Genes Dev. 20: 723.

Zeng H., Qian Z., Myers M.P., and Rosbash M. 1996. A lightentrainment mechanism for the Drosophila circadian clock. Nature 380: 129.

Zerr D.M., Hall J.C., Rosbash M., and Siwicki K.K. 1990. Circadian fluctuations of period protein immunoreactivity in the CNS and the visual system of Drosophila. J. Neurosci. 10: 2749.

Zhao W.N., Malinin N., Yang F.C., Staknis D., Gekakis N., Maier B., Reischl S., Kramer A., and Weitz C.J. 2007. CIPC is a mammalian circadian clock protein without invertebrate homologues. Nat. Cell Biol. 9: 268. 


\section{$\overbrace{\text { SYMPOSI }}^{\infty} \mathrm{CSH}$ Cold Spring Harbor Symposia SYMPOSIA on Quantitative Biology}

\section{Role of Phosphorylation in the Mammalian Circadian Clock}

K. Vanselow and A. Kramer

Cold Spring Harb Symp Quant Biol 2007 72: 167-176

Access the most recent version at doi:10.1101/sqb.2007.72.036

References This article cites 77 articles, 41 of which can be accessed free at: http://symposium.cshlp.org/content/72/167.full.html\#ref-list-1

\section{License}

Email Alerting

Receive free email alerts when new articles cite this article - sign up in the box at the Service top right corner of the article or click here.

To subscribe to Cold Spring Harbor Symposia on Quantitative Biology go to: http://symposium.cshlp.org/subscriptions 